\title{
Approximation of the radiation power of electrons due to the inverse-Compton process in the black-body photon field (Research Note)
}

\author{
O. Petruk \\ Institute for Applied Problems in Mechanics and Mathematics, Naukova St. 3-b, Lviv 79060, Ukraine \\ e-mail: petruk@astro.franko.lviv.ua
}

Received 25 December 2008 / Accepted 25 February 2009

\begin{abstract}
Aims. An approximation is presented for the inverse-Compton radiation power of electrons in the isotropic black-body photon field. Methods. This approximation allows calculation of the inverse-Compton emissivity as an integral over the energies of incident electrons rather than over the field photon energies. Such an approach allows for accurate modeling of IC emission of electrons with energy spectra different from power law, in tasks where essential CPU resources are needed.

Results. The high accuracy of this approximation allows it to be used in a wide range of conditions, from Thomson to extreme Klein-Nishina limits, in different astrophysical objects. This approach also results in some new analytic expressions representing the known results in the Thomson limit.
\end{abstract}

Key words. radiation mechanisms: non-thermal - gamma rays: theory

\section{Introduction}

Inverse Compton (IC) electron-photon interactions are among the most important processes in gamma-ray production in many astrophysical objects. It is (or may be) important in, e.g., diffuse gamma-ray emission from the Galaxy, galaxy clusters, gamma-ray production in supernova remnants (SNRs), pulsarwind nebulae, or in the case of the IC pair cascade processes in the isotropic radiation field, i.e. propagation of gamma-rays over cosmological distances.

Cherenkov detectors open an era of imaging gamma-ray astronomy. Surface brightness distribution of gamma-ray emission of astrophysical objects, in addition to the broad-band spectrum, offers an important possibility of testing models of kinetics of astroparticles, as well as the dynamics of magnetic field and turbulence in astrophysical plasmas. Thus, an important task appears to model the respective gamma-ray maps. Applying the exact formalism of IC emission to such a problem requires, in most cases, essential CPU resources and simulations result therefore simulations therefore lead to very long computations even with the use of modern powerful PC clusters. In such a situation, an accurate approximation may be useful because it considerably reduces CPU time (and also results in some new formulae in classical analysis, see Sects. 2.2 and 2.3 below).

A common approach is to deal with IC emissivity for a given energy of the initial (monochromatic) photons (e.g. Jones 1968; Blumenthal \& Gould 1970) with assumption of a given a priori shape of the electron spectrum, power law as a common choice. The resulting IC photon spectrum is then given by the integration over the energy distribution of the field photons. Such an approach is essential for special cases of the energy distribution of the field photons.
It is known, however, that the conditions when the IC gamma-ray photons are produced by relativistic electrons propagating in the isotropic blackbody radiation field are often met in astrophysical sources. In particular, for SNRs under typical conditions, one may just consider black-body photons, with a few different temperatures representing $\mathrm{CMB} / \mathrm{IR} /$ optical radiation. Even more, in SNRs not assosiated with IR emission, the contribution from CMB photons takes over the role of infrared and optical photons (see discussion in Appendix in Lazendic et al. 2004). The IR/optical photon fields may typically contribute 10-15\% of the IC flux in such SNRs (Gaisser et al. 1998; Baring et al. 1999). Similar situations are found in other astrophysical environments, too.

On the other hand, the shape of the electron spectrum at energies that are important for IC gamma-ray emission may differ considerably from a power law, especially, if one considers the emission of electrons with energy $E$ around maximum possible values.

It is known that contribution from electrons accelerated by the SNR shock to $E_{\max } \sim 30-300 \mathrm{TeV}$ is important for interpreting the HESS observations of shell SNRs. The shape of the end of an electron spectrum is commonly assumed to be maximally curved $N(E)=K E^{-s} \exp \left(-E / E_{\max }\right)$. Some observational and theoretical evidence suggests that the shape is broader around cut-off, $N(E) \propto \exp \left(-\left[E / E_{\max }\right]^{\alpha}\right)$ with $\alpha \approx 0.5$, at least in SN 1006 and G347.3-0.5 (Ellison et al. 2000, 2001; Uchiyama et al. 2003; Lazendic et al. 2004). This broadening is not an artifact of observation, e.g. from superposition of local spectra along the line of sight, but most probably an internal property caused by processes at the acceleration site (Petruk 2006).

Nonlinear acceleration theory predicts the concave shape of the electron spectrum, i.e. varying spectral 
index: $s(p)=2+\delta s(p)$ where $p$ is momentum. The function $\delta s(p)$ monotonically decreases from $\$ 0.2$ at the low-energy end of the spectrum of nonthermal electrons to $\gtrsim-0.5$ around $E_{\max }$ (Berezhko \& Ellison 1999).

Also, the interplay between the synchrotron and IC energy losses can play an essential role in modifying the electron energy spectrum, especially in situations when the ratio of the ambient radiation field to source's magnetic-field energy density, significantly exceeds unity (Moderski et al. 2005). In such a situation, the Klein-Nishina decline may cause the electron spectrum to harden starting from some energy that results in a bump around the cut-off energy. This effect may not be significant for a "typical" SNR, like SN 1006, because the energy density of magnetic field of the strength $B \sim 30 \div 100 \mu \mathrm{G}$ is much higher than the energy density of initial photons. (The magnetic field strength with energy density equal to that in the $\mathrm{CMB}$ is $B=3.27 \mu \mathrm{G}$.) However, for SNRs in radiation-dominated environments (e.g. in the Galactic center), the implications of this effect may be important (Vannoni et al. 2009).

This effect of the Klein-Nishina reduction also influences nonthermal radiation from blazars, micro-quasars, pulsar binaries, and clusters of galaxies (Moderski et al. 2005; Moderski \& Sikora 2005; Vannoni et al. 2009).

In this note, we present an approximation for IC emissivity that may be applied to IC emission originating from the isotropic black-body photon field with temperature $T$. Our approximation is given in terms of an energy of incident electrons rather than in the commonly used terms of the field photon energy. Such an approach gives the possibility of accurately modeling IC emission of electrons with energy spectra different from power law, a situation often appearing in astrophysical objects.

Since the temperature of the initial photons $T$ is a parameter in our approach, our approximation may be used for calculating IC radiation from different photon fields (CMB, IR, optical). The target radiation field in some circumstances (e.g. around Galactic center) may not be black-body and/or the contribution from IR/optical photons may dominate CMB there (Porter et al. 2006; Hinton \& Aharonian 2007). In cases where the different components of the target radiation field may be approached by a superposition of multiple Planck distributions with different $T$, our approximation may be used in a similar fashion. The overall IC emission will be the weighted sum of single approximations, each with a different value for the temperature. In cases where the initial radiation field may not be approximately described by a sum of black-body distributions, our formulae do not apply.

Another assumption in the present paper is the isotropy of the electron and photon fields. A thorough treatment of anisotropic IC scattering from cosmic-ray electrons is done by Moskalenko \& Strong (2000).

\section{Approximation}

The spectral distribution of the volume emissivity of (isotropically distributed) electrons due to the IC process is (Jones 1968; Blumenthal \& Gould 1970; Schlickeiser 2002)

$P\left(E_{\gamma}\right)=c E_{\gamma} \int \mathrm{d} \gamma N(\gamma) \int \mathrm{d} \epsilon n_{\mathrm{ph}}(\epsilon) \sigma_{\mathrm{KN}}\left(E_{\gamma}, \epsilon ; \gamma\right)$

where $\gamma$ is the Lorenz factor of the electron, $N(\gamma)$ the spectral distribution of electrons, $\epsilon$ and $E_{\gamma}$ are the energies of photon before and after interaction, $n_{\mathrm{ph}}(\epsilon)$ is the (isotropic) initial photon energy distribution,

$\sigma_{\mathrm{KN}}\left(E_{\gamma}, \epsilon ; \gamma\right)=\frac{3 \sigma_{\mathrm{T}}}{4 \epsilon \gamma^{2}} G(q, \eta)$ is the angle-integrated IC cross-section, $\sigma_{\mathrm{T}}$ the Thomson cross-section,

$G(q, \eta)=2 q \ln q+(1+2 q)(1-q)+2 \eta q(1-q)$,

and

$q=\frac{E_{\gamma}}{\Gamma\left(\gamma m_{\mathrm{e}} c^{2}-E_{\gamma}\right)}, \quad \Gamma=\frac{4 \epsilon \gamma}{m_{\mathrm{e}} c^{2}}, \quad \eta=\frac{\epsilon E_{\gamma}}{\left(m_{\mathrm{e}} c^{2}\right)^{2}}$.

The last term in $G$ reflects the Klein-Nishina decline; recoil is embedded in the $q$ parameter. The $\mathrm{KN}$ effect is more important than recoil for $\Gamma \geq 1$ (e.g. Baring et al. 1999). Kinematic requirements result in $\left(4 \gamma^{2}\right)^{-1} \leq q \leq 1$ (Blumenthal \& Gould 1970). Setting $q$ to its minimum and maximum values limits energies of up-scattered photons:

$E_{\gamma, \min }=\frac{\gamma m_{\mathrm{e}} c^{2} \Gamma}{4 \gamma^{2}+\Gamma}$

$E_{\gamma, \max }=\frac{\gamma m_{\mathrm{e}} c^{2} \Gamma}{1+\Gamma}$

that simplifies to

$E_{\gamma, \min }=\epsilon, \quad E_{\gamma, \max }=4 \gamma^{2} \epsilon$

in Thomson limit $(\Gamma \ll 1)$ and to

$E_{\gamma, \min }=\epsilon\left(\right.$ if $\left.\Gamma \ll 4 \gamma^{2}\right), \quad E_{\gamma, \max }=\gamma m_{\mathrm{e}} c^{2}$

in extreme Klein-Nishina limit $(\Gamma \gg 1)$. The condition $q \leq 1$ sets the minimum Lorentz factor

$\gamma_{\text {min }}=\frac{E_{\gamma}}{2 m_{\mathrm{e}} c^{2}}\left[1+\left(1+\frac{\left(m_{\mathrm{e}} c^{2}\right)^{2}}{\epsilon E_{\gamma}}\right)^{1 / 2}\right]$

electron should have in order to scatter photon with energy $\epsilon$ to energy $E_{\gamma}$. The function $\gamma_{\min }\left(E_{\gamma}\right)$ may approximately be split into two parts

$\gamma_{\min }= \begin{cases}E_{\gamma}^{1 / 2} /\left(2 \epsilon^{1 / 2}\right), & \text { for } \quad \eta \ll 1 \\ E_{\gamma} /\left(m_{\mathrm{e}} c^{2}\right), & \text { for } \eta \gg 1\end{cases}$

(The Klein-Nishina decline is negligible for $\eta \ll 1$, Eq. (3).) The point where one could approximately switch from $\gamma_{\min } \propto E_{\gamma}^{1 / 2}$ to $\gamma_{\min } \propto E_{\gamma}$ is

$E_{\gamma, *}=\frac{\left(m_{\mathrm{e}} c^{2}\right)^{2}}{4 \epsilon}$

\subsection{Method of approximation}

In some astrophysical environments, the initial photon field may well be represented by the isotropic black-body radiation

$n_{\mathrm{ph}}(\epsilon)=\frac{1}{\pi^{2} \hbar^{3} c^{3}} \frac{\epsilon^{2}}{\exp \left(\epsilon / \epsilon_{\mathrm{c}}\right)-1}$

with $\epsilon_{\mathrm{c}}=k T$.

Let us re-write Eq. (1) in the form

$P\left(E_{\gamma}\right)=\int \mathrm{d} \gamma N(\gamma) p\left(\gamma, E_{\gamma}\right)$ 
where the spectral distribution of IC radiation power of a "single" electron with Lorenz factor $\gamma$ is

$p\left(\gamma, E_{\gamma}\right)=\frac{3 \sigma_{\mathrm{T}} m_{\mathrm{e}}^{2} c^{2} \epsilon_{\mathrm{c}}}{4 \pi^{2} \hbar^{3}} \gamma^{-2} \mathcal{I}\left(\eta_{\mathrm{c}}, \eta_{\mathrm{o}}\right)=\frac{2 e^{4} \epsilon_{\mathrm{c}}}{\pi \hbar^{3} c^{2}} \gamma^{-2} \mathcal{I}\left(\eta_{\mathrm{c}}, \eta_{\mathrm{o}}\right)$

with the function $\mathcal{I}\left(\eta_{\mathrm{c}}\left(E_{\gamma}\right), \eta_{\mathrm{o}}\left(\gamma, E_{\gamma}\right)\right)$

$\mathcal{I}\left(\eta_{\mathrm{c}}, \eta_{\mathrm{o}}\right)=\int \frac{\left(\eta / \eta_{\mathrm{c}}\right) G\left(\eta_{\mathrm{o}} / \eta, \eta\right)}{\exp \left(\eta / \eta_{\mathrm{c}}\right)-1} \mathrm{~d} \eta$

$\eta_{\mathrm{c}}=\frac{\epsilon_{\mathrm{c}} E_{\gamma}}{\left(m_{\mathrm{e}} c^{2}\right)^{2}}, \quad \eta_{\mathrm{o}} \equiv q \eta=\frac{E_{\gamma}^{2}}{4 \gamma m_{\mathrm{e}} c^{2}\left(\gamma m_{\mathrm{e}} c^{2}-E_{\gamma}\right)}$.

Let us introduce

$G_{1}(q)=2 q \ln q+(1+2 q)(1-q)$,

$G_{2}\left(q, \eta_{\mathrm{o}}\right)=2 \eta_{\mathrm{o}}(1-q)$.

In the limit $\eta \rightarrow \infty, G_{1}, G_{2}, G$ asymptotically approach the values

$G_{1, \mathrm{as}}=1, \quad G_{2, \mathrm{as}}=2 \eta_{\mathrm{o}}, \quad G_{\mathrm{as}}=1+2 \eta_{\mathrm{o}}$.

The minimum value of $\eta$, namely $\eta_{\min }=\eta_{\mathrm{o}}$, is given by the condition $G\left(\eta_{\mathrm{o}} / \eta, \eta_{\mathrm{o}}\right)=0$. Relation $\eta_{\min }=\eta_{\mathrm{o}}$ with definition of $\eta$, Eq. (4), yield the formula for the minimum energy of the photon $\epsilon_{\min }$, which may be up-scattered to the energy $E_{\gamma}$ by the electron with Lorentz factor $\gamma$ :

$\epsilon_{\min }=\frac{E_{\gamma} m_{\mathrm{e}} c^{2}}{4 \gamma\left(\gamma m_{\mathrm{e}} c^{2}-E_{\gamma}\right)}$.

In the limit $\eta_{\mathrm{o}} \ll \eta_{\mathrm{c}}$, which is equivalent to the Thomson limit $\Gamma\left(\epsilon_{\mathrm{c}}\right) \ll 1$, the integral (15) may be found analytically

$\mathcal{I}_{\mathrm{T}}\left(\eta_{\mathrm{c}}, \eta_{\mathrm{o}}\right)=\eta_{\mathrm{c}} \int_{0}^{\infty} \frac{\eta^{\prime} \mathrm{d} \eta^{\prime}}{\exp \left(\eta^{\prime}\right)-1}=\frac{\pi^{2} \eta_{\mathrm{c}}}{6}$.

With decreasing $\eta, G$ falls rather rapidly from $G_{\text {as }}$ to zero. Let $G\left(\eta_{\mathrm{o}} / \eta, \eta\right)$ be approximated by Heavicide step function

$G\left(\eta_{\mathrm{o}} / \eta, \eta\right) \approx\left(1+2 \eta_{\mathrm{o}}\right) H\left(\eta-\eta_{\mathrm{o}}\right)$.

The integral $\mathcal{I}$ may then be approximately found as $\mathcal{I}\left(\eta_{\mathrm{c}}, \eta_{\mathrm{o}}\right) \approx$ $\mathcal{I}_{\mathrm{H}}\left(\eta_{\mathrm{c}}, \eta_{\mathrm{o}}\right)$ :

$$
\begin{aligned}
\mathcal{I}_{\mathrm{H}}\left(\eta_{\mathrm{c}}, \eta_{\mathrm{o}}\right) & =\left(1+2 \eta_{\mathrm{o}}\right) \int_{\eta_{\mathrm{o}}}^{\infty} \frac{\eta / \eta_{\mathrm{c}}}{\exp \left(\eta / \eta_{\mathrm{c}}\right)-1} \mathrm{~d} \eta \\
& =\left(1+2 \eta_{\mathrm{o}}\right) \eta_{\mathrm{c}}\left(\frac{\pi^{2}}{6}+\operatorname{Li}_{2}\left(\exp \left(\eta_{\mathrm{o}} / \eta_{\mathrm{c}}\right)\right)+\frac{\left(\eta_{\mathrm{o}} / \eta_{\mathrm{c}}\right)^{2}}{2}\right),
\end{aligned}
$$

where the dilogarithm function

$\operatorname{Li}_{2}(x)=-\int_{x}^{1} \frac{\ln (t)}{1-t} \mathrm{~d} t$

An accurate approximation of (23) is

$\mathcal{I}_{\mathrm{H}}\left(\eta_{\mathrm{c}}, \eta_{\mathrm{o}}\right) \approx \frac{\pi^{2}}{6} \eta_{\mathrm{c}}\left(1+2 \eta_{\mathrm{o}}\right) \exp \left(-\frac{2 \eta_{\mathrm{o}}}{3 \eta_{\mathrm{c}}}\right)$.
This expression restores $\mathcal{I}$ almost exactly for $\eta_{\mathrm{o}} / \eta_{\mathrm{c}} \lesssim 10^{-2}$ and $\eta_{\mathrm{o}} / \eta_{\mathrm{c}} \gtrsim 1$. However, $\mathcal{I}_{\mathrm{H}}\left(\eta_{\mathrm{c}}, \eta_{\mathrm{o}}\right)$ overestimates $\mathcal{I}\left(\eta_{\mathrm{c}}, \eta_{\mathrm{o}}\right)$ up to 2 times for $10^{-2} \lesssim \eta_{\mathrm{o}} / \eta_{\mathrm{c}} \lesssim 1$. This is because the deviation of $G$ from the Heavicide step function is important in this range.

The original integral $I$ has an important property, because it may be split as $\mathcal{I}=\mathcal{I}_{1}+\mathcal{I}_{2}$ with

$$
\begin{aligned}
\mathcal{I}_{1}\left(\eta_{\mathrm{c}}, \eta_{\mathrm{o}}\right) & =\int \frac{\left(\eta / \eta_{\mathrm{c}}\right) G_{1}\left(\eta_{\mathrm{o}} / \eta\right)}{\exp \left(\eta / \eta_{\mathrm{c}}\right)-1} \mathrm{~d} \eta=\eta_{\mathrm{c}} \int \frac{x G_{1}\left(x_{\mathrm{o}} / x\right)}{\exp (x)-1} \mathrm{~d} x \\
\mathcal{I}_{2}\left(\eta_{\mathrm{c}}, \eta_{\mathrm{o}}\right) & =\eta_{\mathrm{o}} \int \frac{\left(\eta / \eta_{\mathrm{c}}\right) G_{2 *}\left(\eta_{\mathrm{o}} / \eta\right)}{\exp \left(\eta / \eta_{\mathrm{c}}\right)-1} \mathrm{~d} \eta \\
& =\eta_{\mathrm{c}} \eta_{\mathrm{o}} \int \frac{x G_{2 *}\left(x_{\mathrm{o}} / x\right)}{\exp (x)-1} \mathrm{~d} x
\end{aligned}
$$

where $x_{\mathrm{o}}=\eta_{\mathrm{o}} / \eta_{\mathrm{c}}$ and $G_{2 *}=G_{2} / \eta_{\mathrm{o}}$. As one can see, these integrals may be scaled:

$\mathcal{I}_{1}\left(a \eta_{\mathrm{c}}, a \eta_{\mathrm{o}}\right)=a \mathcal{I}_{1}\left(\eta_{\mathrm{c}}, \eta_{\mathrm{o}}\right), \quad \mathcal{I}_{2}\left(a \eta_{\mathrm{c}}, a \eta_{\mathrm{o}}\right)=a^{2} \mathcal{I}_{2}\left(\eta_{\mathrm{c}}, \eta_{\mathrm{o}}\right)$

The possibility of scaling these integrands (integral $I$ may not be universally scaled) is an important property that allows us to obtain an analytic approximation. It is also the reason for the approximation accuracy over the wide range of parameters.

Using $a=\eta_{\mathrm{c}}^{-1}$ in the scale laws (28), one has

$\mathcal{I}_{1}\left(\eta_{\mathrm{c}}, \eta_{\mathrm{o}}\right)=\eta_{\mathrm{c}} \mathcal{I}_{1}\left(1, \eta_{\mathrm{o}} / \eta_{\mathrm{c}}\right), \quad \mathcal{I}_{2}\left(\eta_{\mathrm{c}}, \eta_{\mathrm{o}}\right)=\eta_{\mathrm{c}}^{2} \mathcal{I}_{2}\left(1, \eta_{\mathrm{o}} / \eta_{\mathrm{c}}\right)$.

This means that it is enough to check how accurate approximate expressions will be in approximation of just $\mathcal{I}_{1}(1, x)$ and $I_{2}(1, x)$, and we will know how accurate these approximations will be for any $\eta_{\mathrm{c}}$ and $\eta_{\mathrm{o}}$.

Let us slightly correct exponential part in each terms of (25) by introducing into the exponents the second terms of the form $c_{1}\left(\eta_{\mathrm{o}} / \eta_{\mathrm{c}}\right)^{c_{2}}$ where $c_{1}$ and $c_{2}$ are constant. These terms make (25) more accurate in the representation of $\mathcal{I}_{1}$ and $\mathcal{I}_{2}$. It is important that the terms are also scaled with (28). With these corrections, we come to approximations

$\mathcal{I}_{1} \approx \frac{\pi^{2}}{6} \eta_{\mathrm{c}} \exp \left[-\frac{2 \eta_{\mathrm{o}}}{3 \eta_{\mathrm{c}}}-\frac{5}{4}\left(\frac{\eta_{\mathrm{o}}}{\eta_{\mathrm{c}}}\right)^{1 / 2}\right]$

$\mathcal{I}_{2} \approx \frac{\pi^{2}}{3} \eta_{\mathrm{c}} \eta_{\mathrm{o}} \exp \left[-\frac{2 \eta_{\mathrm{o}}}{3 \eta_{\mathrm{c}}}-\frac{5}{7}\left(\frac{\eta_{\mathrm{o}}}{\eta_{\mathrm{c}}}\right)^{0.7}\right]$

The values of $c_{1}$ and $c_{2}$ are obtained by fitting the exact $I_{1}(1, x)$ and $I_{2}(1, x)$. The approximations are compared with the exact dependences in Fig. 1 which reveals good accuracy of obtained approximate formulae.

The scaling property is a reason that the sum of approximations (30) and (31)

$$
\begin{aligned}
\mathcal{I}\left(\eta_{\mathrm{c}}, \eta_{\mathrm{o}}\right) \approx & \frac{\pi^{2}}{6} \eta_{\mathrm{c}}\left(\exp \left[-\frac{5}{4}\left(\frac{\eta_{\mathrm{o}}}{\eta_{\mathrm{c}}}\right)^{1 / 2}\right]\right. \\
& \left.+2 \eta_{\mathrm{o}} \exp \left[-\frac{5}{7}\left(\frac{\eta_{\mathrm{o}}}{\eta_{\mathrm{c}}}\right)^{0.7}\right]\right) \exp \left[-\frac{2 \eta_{\mathrm{o}}}{3 \eta_{\mathrm{c}}}\right]
\end{aligned}
$$

is accurate to represent $\mathcal{I}$ in any regime, from Thomson to extreme Klein-Nishina. 


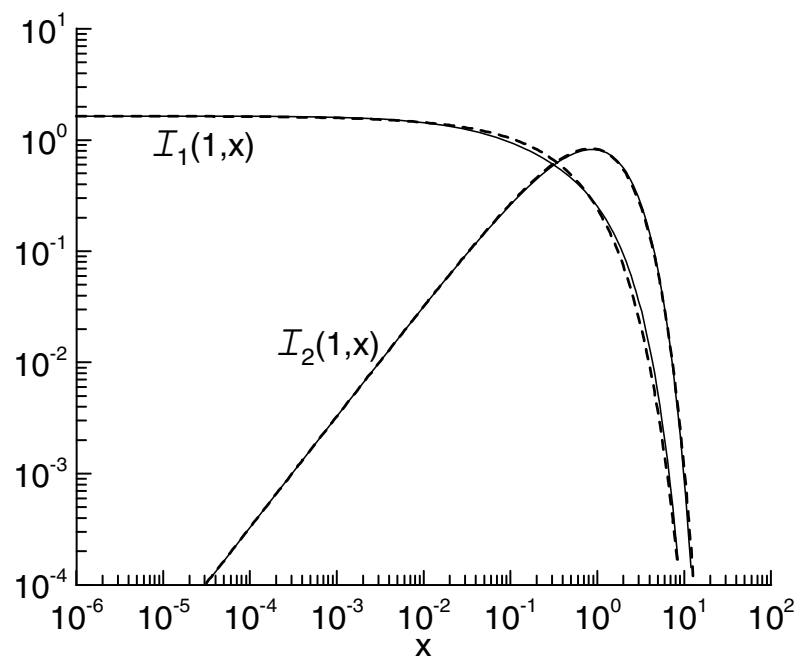

Fig. 1. The accuracy of the approximation (32) as the sum $I_{1}+I_{2}$. Integrals $\mathcal{I}_{1}(1, x), \mathcal{I}_{2}(1, x)$ (solid lines) are compared here with their approximations (30) and (31) (dotted lines).

Equation (32) is good for any $\eta_{\mathrm{c}}$. We may suggest two simpler approximations for different ranges of $\eta_{\mathrm{c}}$. If mostly interested in $\eta_{\mathrm{c}} \lesssim 100$ (e.g. the case of IC emission of electrons accelerated by the forward shock in SNRs), then one can use an expression

$\mathcal{I}\left(\eta_{\mathrm{c}}, \eta_{\mathrm{o}}\right) \approx \frac{\pi^{2}}{6} \eta_{\mathrm{c}}\left(1+3 \eta_{\mathrm{o}}\right) \exp \left[-\frac{2 \eta_{\mathrm{o}}}{3 \eta_{\mathrm{c}}}-\frac{5}{4}\left(\frac{\eta_{\mathrm{o}}}{\eta_{\mathrm{c}}}\right)^{1 / 2}\right]$.

Figure 2 shows that the second term here (the one proportional to $3 \eta_{\mathrm{o}}$ ) overestimates $\mathcal{I}_{2}$ in its power-law part. Nevertheless, this error is negligible for $\eta_{\mathrm{c}} \leq 100$ (Fig. 2). If $\eta_{\mathrm{c}} \gtrsim 10$ is of interest, then one may neglect accuracy in exponential part of approximation of $\mathcal{I}_{1}$ and use the approximation (Fig. 3)

$\mathcal{I}\left(\eta_{\mathrm{c}}, \eta_{\mathrm{o}}\right) \approx \frac{\pi^{2}}{6} \eta_{\mathrm{c}}\left(1+2 \eta_{\mathrm{o}}\right) \exp \left[-\frac{2 \eta_{\mathrm{o}}}{3 \eta_{\mathrm{c}}}-\frac{5}{7}\left(\frac{\eta_{\mathrm{o}}}{\eta_{\mathrm{c}}}\right)^{0.7}\right]$.

Figure 4 shows the accuracy of Eq. (33) in approximation of emission power (14) for electrons with energies $10^{12} \div 10^{16} \mathrm{eV}$.

\section{2. "Delta-function" approximation}

Figure 4 demonstrates that a "single" electron with Lorentz factor $\gamma$ - being scattered by all black-body photons - emits most of its IC radiation at photons with some characteristic energy $E_{\gamma \mathrm{m}}$. One can introduce a "delta-function approximation", in addition to the classical "monochromatic approximation" where the electron is scattered by the photons with a fixed energy $\epsilon_{\mathrm{o}}$ (e.g. Schlickeiser 2002). One can namely assume that a "single" electron (scattered by all black-body photons) emits all of its IC power at photons with $E_{\gamma \mathrm{m}}$ :

$p\left(\gamma, E_{\gamma}\right) \approx p_{\mathrm{m}}(\gamma) \delta\left(E_{\gamma}-E_{\gamma \mathrm{m}}\right)$

where

$p_{\mathrm{m}}(\gamma)=\int_{0}^{\infty} p\left(\gamma, E_{\gamma}\right) \mathrm{d} E_{\gamma}$.

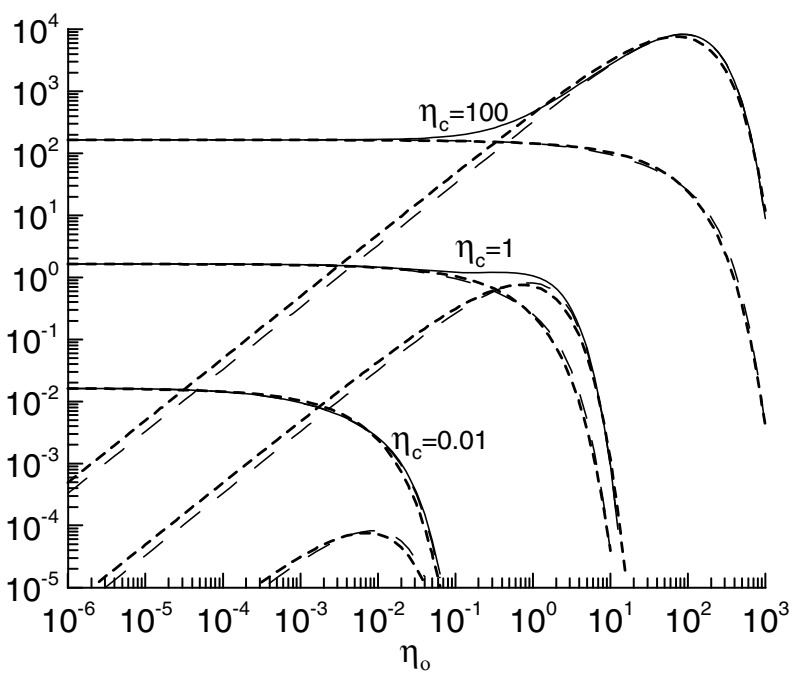

Fig. 2. Accuracy of approximation (33). Integrals $\mathcal{I}$ (15) (solid lines), $I_{1}$ and $I_{2}$ (26), (27) (dashed lines), and respective terms of approximation (33) (dotted lines) versus $\eta_{\mathrm{o}}$ for a number of $\eta_{\mathrm{c}}=0.01,1,100$ (from below).

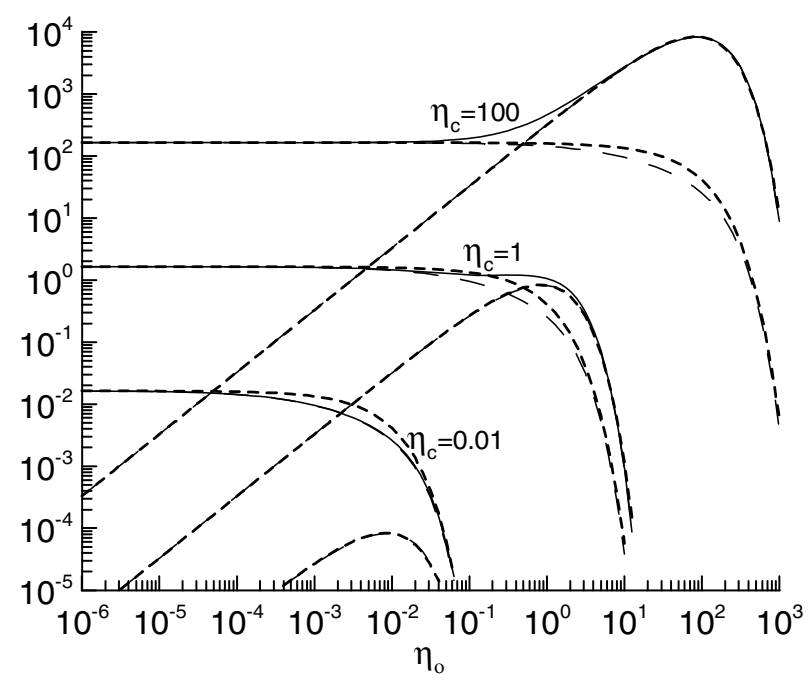

Fig. 3. Accuracy of approximation (34). Integrals $\mathcal{I}$ (15) (solid lines), $\mathcal{I}_{1}$ and $\mathcal{I}_{2}$ (26), (27) (dashed lines), and respective summands of approximation (34) (dotted lines) versus $\eta_{\mathrm{o}}$ for a number of $\eta_{\mathrm{c}}=$ $0.01,1,100$ (from below).

There are well known approximations for the total IC energy loss $p_{\mathrm{m}}$ of electron in the Thomson (see (41) below) and extreme Klein-Nishina limits (e.g. Sect. 4.2.3 in Schlickeiser 2002).

Our numerical calculations show (Fig. 4) that $E_{\gamma \mathrm{m}}$ may be approximated by

$E_{\gamma \mathrm{m}}(\gamma) \approx E_{\gamma, \max }\left(\gamma, \epsilon_{\mathrm{c}}\right)$

where $E_{\gamma, \max }(\gamma, \epsilon)$ is given by (6). In the Thomson limit, this is

$E_{\gamma \mathrm{m}}(\gamma) \approx 4 \epsilon_{\mathrm{c}} \gamma^{2}$

In the classical "monochromatic approximation" (e.g. Schlickeiser 2002), the average $\left\langle E_{\gamma}\right\rangle=(4 / 3) \epsilon_{0} \gamma^{2}$ is used to estimate the energy of "monochromatic" IC photons emitted by electron. 


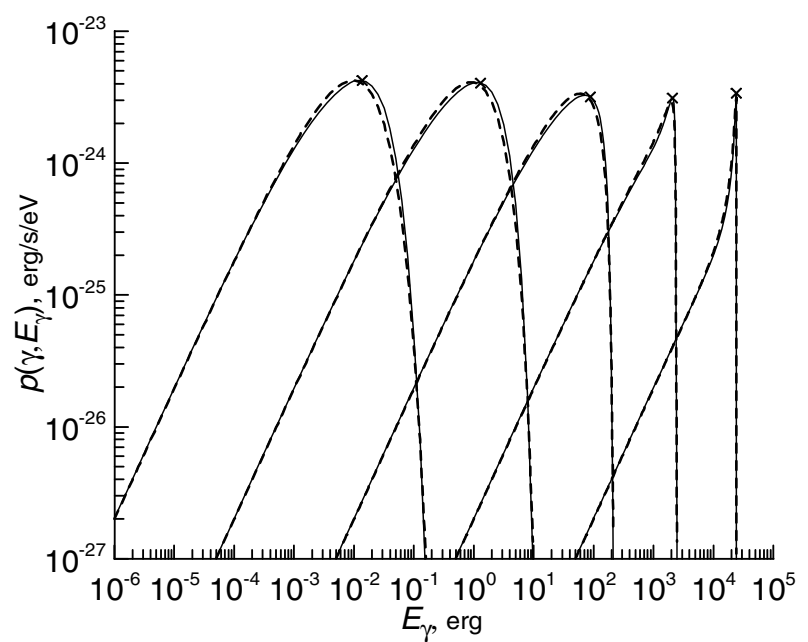

Fig. 4. The spectrum $p\left(E_{\gamma}\right)(14)$ calculated with integral $I$ (solid lines) and with its approximation (33) (dashed lines) for energies of electrons $E=1.5 \times\left(10^{12}, 10^{13}, 10^{14}, 10^{15}, 10^{16}\right) \mathrm{eV}$. Crosses correspond to position of $E_{\gamma, \max }\left(\gamma, \epsilon_{\mathrm{c}}\right)$. The temperature of the black-body radiation is $T=2.75 \mathrm{~K}$.

\subsection{Thomson limit}

The use of (21) in (14) allows us to write down the expression for IC emissivity in the Thomson limit $\eta_{\mathrm{o}} \ll \eta_{\mathrm{c}}$. The spectral distribution of IC radiation power of a "single" electron with energy $E=\gamma m_{\mathrm{e}} c^{2}$ is

$p_{\mathrm{T}}\left(\gamma, E_{\gamma}\right)=\frac{\sigma_{\mathrm{T}} \epsilon_{\mathrm{c}}^{2}}{8 \hbar^{3} c^{2}} \frac{E_{\gamma}}{\gamma^{2}}=\frac{\pi e^{4} \epsilon_{\mathrm{c}}^{2}}{3 \hbar^{3} c^{2}} \frac{E_{\gamma}}{E^{2}}, \quad E_{\gamma} \leq E_{\gamma, \mathrm{lim}}$

where $E_{\gamma, \text { lim }}$ is the characteristic maximum energy defined below. This expression represents integration over all possible energies $\epsilon$ of the seed black-body photons.

The power $p_{\mathrm{T}}\left(\gamma, E_{\gamma}\right)$ is the increasing function of $E_{\gamma}$, while $p\left(\gamma, E_{\gamma}\right)$ decreases rather rapidly after the maximum (Fig. 4). We define an energy $E_{\gamma, \text { lim }}$ by the condition

$$
\int_{0}^{E_{\gamma, \lim }} p_{\mathrm{T}}\left(\gamma, E_{\gamma}\right) \mathrm{d} E_{\gamma}=p_{\mathrm{mT}}(\gamma)
$$

where

$p_{\mathrm{mT}}(\gamma)=(4 / 3) c \sigma_{\mathrm{T}} \omega \gamma^{2}$

is the total energy loss of electron due to IC in the Thomson limit, and $\omega=\int \epsilon n_{\mathrm{ph}}(\epsilon) \mathrm{d} \epsilon$ is the energy density of all initial photons. The definition (40) results in

$E_{\gamma, \lim }=\frac{4}{\pi} \epsilon_{\mathrm{c}} \gamma^{2}\left[\frac{2}{3} \int_{0}^{\infty} \frac{z^{3} \mathrm{~d} z}{\exp (z)-1}\right]^{1 / 2}=2.65 \epsilon_{\mathrm{c}} \gamma^{2}$.

Note that $E_{\gamma, \text { lim }}$ differs only a little from $E_{\gamma, \max }\left(\epsilon_{\mathrm{c}}\right)=4 \epsilon_{\mathrm{c}} \gamma^{2}$.

The volume emissivity of electrons distributed as

$N(\gamma)=N_{\mathrm{o}} \gamma^{-s}$ for $\quad \gamma_{\min }<\gamma<\gamma_{\max }, \quad \gamma_{\min } \ll \gamma_{\max }$

is

$P\left(E_{\gamma}\right)=\frac{\sigma_{\mathrm{T}} \epsilon_{\mathrm{c}}^{2}}{8 \hbar^{3} c^{2}(s+1)} N_{\mathrm{o}} E_{\gamma} \gamma_{\min }^{-(s+1)}$.
For $E_{\gamma} \lesssim E_{\gamma, *}$, the minimum Lorentz factor is $\gamma_{\min }=$ $E_{\gamma}^{1 / 2} /\left(2 \epsilon_{*}^{1 / 2}\right)$, Eq. (10), and we approximate the (13) in the Thomson limit

$P\left(E_{\gamma}\right)=\frac{2^{s-2} \sigma_{\mathrm{T}} \epsilon_{\mathrm{c}}^{2} \epsilon_{*}^{(s+1) / 2}}{\hbar^{3} c^{2}(s+1)} N_{\mathrm{o}} E_{\gamma}^{-(s-1) / 2}$,

with the known slope $P\left(E_{\gamma}\right) \propto E_{\gamma}^{-(s-1) / 2}$. The value of $\epsilon_{*}$ may be fixed by comparing (45) with e.g. expression (4.2.17) in Schlickeiser (2002); i.e., $\epsilon_{*}=\mathcal{A}(s) \epsilon_{\mathrm{c}}$ with

$\mathcal{A}(s)=\left[\frac{12}{\pi^{2}} \frac{\left(s^{2}+4 s+11\right)}{(s+5)(s+3)^{2}} \int_{0}^{\infty} \frac{z^{(s+3) / 2} \mathrm{~d} z}{\exp (z)-1}\right]^{2 /(s+1)}$.

Numerically, $\mathcal{A}(1.8)=0.665, \mathcal{A}(2)=0.710, \mathcal{A}(2.2)=0.755$.

\section{Conclusions}

Numerical evaluation of the spatial distribution of the IC emission (e.g. in SNRs) requires essential computational resources because the IC volume emissivity (1) consists in two enclosed integrations (over initial photon and electron energies).

We developed the approximation (32) of the spectral distribution of the IC emission power $p\left(E_{\gamma}\right)$ of electrons with Lorentz factor $\gamma$, which are interacting with the isotropic blackbody photon field. Equation (32) restores the known results (Blumenthal \& Gould 1970) with sufficient accuracy in any regime, from Thomson to extreme Klein-Nishina limits. It may be used for different astrophysical objects, from pulsar wind nebulae to clusters of galaxies. For $k T E_{\gamma} \lesssim 100\left(m_{\mathrm{e}} c^{2}\right)^{2}$, e.g. for IC $\gamma$-ray emission from electrons accelerated by the forward shock of SNRs, it is suitable to use a simpler approximation (33). In the Thomson limit, our approach results in a simple expression (39).

Our approximation may be used in situations where the initial radiation field may be approximated with the Planck function with some temperature $T$ or when it may be represented by a superposition of the black-body distributions with different $T$. In addition, it assumes the isotropy of electron and photon fields.

The approximation is given in terms of an energy of incident electrons rather than in terms of the field photon energy. (There are known approximations for the latter approach, see e.g. Schlickeiser 2002.) Therefore, our approximation may be useful for analysing the role of the electron spectrum with shapes that differ from the power law.

The main idea behind our approach is the possibility of splitting the initial integral into two parts that may be scaled, contrary to the original integral. This scaling is the reason for the accuracy of the approximation over the wide range of parameters, from the Thomson to extreme Klein-Nishina regime.

There is a well known "monochromatic approximation" where an electron is scattered by the monochromatic photons with energy $\epsilon_{\mathrm{o}}$ (e.g. Schlickeiser 2002). Figure 4 shows that the spectral distribution of the radiation power of a "single" electron with Lorentz factor $\gamma$ scattered by photons distributed with the Planck function peaks at energy $E_{\gamma \mathrm{m}}(\gamma)$. This allows us to introduce - as for the case of synchrotron emission - the "deltafunction" approximation for IC emission. In this approximation, Eq. (35), all radiated energy of electron is assumed to be at $E_{\gamma \mathrm{m}}(\gamma)$. In the Thomson limit, $E_{\gamma \mathrm{m}}(\gamma) \approx 4 \epsilon_{\mathrm{c}} \gamma^{2}$ where $\epsilon_{\mathrm{c}}=k T$. In the classical "monochromatic approximation" (where electrons meet monochromatic photons with energy $\epsilon_{\mathrm{o}}$ ), the average 
$\left\langle E_{\gamma}\right\rangle=(4 / 3) \epsilon_{\mathrm{o}} \gamma^{2}$ is used as an estimator for the energy of emitted IC photons.

Our approach results in some new expressions that represent known results. Equation (39) yields the spectral distribution of IC radiation power of a "single" electron and Eq. (45) represents the spectrum of IC emission from the power-law spectrum of electrons in the Thomson limit. These expression account for integration over all possible energies of the seed black-body photons. They can be derived thanks to the possibility of analytical integration of $\mathcal{I}$ in the Thomson regime, Eq. (21).

\section{References}

Baring, M. G., Ellison, D. C., Reynolds, S. P., Grenier, I. A., \& Goret, P. 1999, ApJ, 513, 311
Berezhko, E., \& Ellison, D. 1999, ApJ, 526, 385

Blumenthal, G., \& Gould, R. 1970, Rev. Mod. Phys., 42, 237

Ellison, D. C., Berezhko, E. G., \& Baring, M. G. 2000, ApJ, 540, 292

Ellison, D. C., Slane, P., \& Gaensler, B. M. 2001, ApJ, 563, 191

Gaisser, T. K., Protheroe, R. J., \& Stanev, T. 1998, ApJ, 492, 219

Hinton, J. A., \& Aharonian, F. A. 2007, ApJ, 657, 302

Jones, F. 1968, Phys. Rev., 167, 1159

Lazendic, J. S., Slane, P. O., Gaensler, B. M., et al. 2004, ApJ, 602, 271

Moderski, R., \& Sikora, M. 2005, Ap\&SS, 297, 369

Moderski, R., Sikora, M., Coppi, P. S., \& Aharonian, F. 2005, MNRAS, 363, 954

Moskalenko, I. V., \& Strong, A. W. 2000, ApJ, 528, 357

Petruk, O. 2006, A\&A, 460, 375

Porter, T. A., Moskalenko, I. V., \& Strong, A. W. 2006, ApJ, 648, L29

Schlickeiser, R. 2002, Cosmic Ray Astrophysics (Springer)

Uchiyama, Y., Aharonian, F. A., \& Takahashi, T. 2003, A\&A, 400, 567

Vannoni, G., Gabici, S., \& Aharonian, F. 2009, A\&A, 497, 17 